\title{
石墨烯在甘油/尿素剥离液中的稳定行为的分子动力学模拟研究
}

\author{
刘长安 $a, b \quad$ 洪士博 $c$ 李蓓*,a,b \\ ( ${ }^{a}$ 武汉理工大学材料科学与工程学院 武汉 430070) \\ ( $b$ 武汉理工大学材料基因工程研究中心 武汉 430070) \\ $\left({ }^{c}\right.$ 重庆国际复合材料股份有限公司 重庆 400082)
}

\begin{abstract}
摘要 现有的实验方法很难实时观测到石墨烯在液相剥离溶剂中的结构演变, 尤其是石墨烯稳定的微观机理尚不明 确. 本工作通过分子动力学方法, 模拟了多层石墨烯和 U 型石墨烯在不同的物质的量比下的甘油/尿素溶剂中的结构变 化, 研究剥离液对石墨烯稳定性的影响. 结果表明, 多层石墨烯在不同溶剂体系中的稳定性差异不显著; 而 U 型石墨 烯在各溶剂体系的稳定性有明显差异, 且稳定能力为: 纯甘油 $>$ 甘油/尿素(2/1)> 甘油/尿素 $(3 / 1)>$ 甘油/尿素 $(1 / 1)$. 这说 明石墨烯在剥离溶剂中的稳定性与石墨烯的剥离状态有关. 通过溶剂分布发现, 尿素能够进入石墨烯层间, 增加石墨 烯层间距; 同时，甘油能够与尿素形成氢键，随尿素进入石墨烯层间，进一步增大层间距，从而形成稳定的单层或多层 受限二元溶剂分子层. 受限溶剂分子层对剥离的石墨烯存在排斥作用, 从而为石墨烯在甘油/尿素二元剥离液中的长期 稳定提供了保障.
\end{abstract}

关键词 分子动力学; 稳定性; 石墨烯; 甘油; 尿素

\section{Molecular Dynamics Simulation of the Stability Behavior of Graphene in Glycerol/Urea Solvents in Liquid-Phase Exfoliation}

\author{
Chang-An Liu ${ }^{a, b}$ \\ Shi-Bo Hong ${ }^{c}$ \\ Bei $\mathrm{Li}^{*, a, b}$ \\ ( ${ }^{a}$ School of Materials Science and Engineering, Wuhan University of Technology, Wuhan 430070, China) \\ ( ${ }^{b}$ Research Center for Materials Genome Engineering, Wuhan University of Technology, Wuhan 430070, China) \\ ( ${ }^{c}$ Chongqing Polycomp International Corporation, Chongqing 400082, China)
}

\begin{abstract}
Understanding of interfacial structure and stabilization mechanism of graphene sheets in green solvents during liquid-phase exfoliation is of great importance in advancing preparation, characterization and synthesis of graphene-based materials. However, it is difficult to monitor structural evolution of graphene in solvents using current available experimental techniques, and the resulting graphene stabilization mechanism has not been fully understood. In this work, molecular dynamics simulations are performed to investigate the structural evolution and stability behavior of the graphene sheets with different states in the glycerol/urea green solvents with varying concentrations. The results show that only the pristine graphene sheet at an initial interlayer spacing of $0.6 \mathrm{~nm}$ in the glycerol solvent restacks back and stays close to each other at a separation close to the intrinsic thickness of graphene. This is due to the fact that the glycerol molecules fail to diffuse into the graphene interlayer, and they could not afford a sufficient repulsive barrier to hinder the graphene aggregation. While in other pristine cases, a single- or double-layer solvent structure is formed and the interlayer separation is maintained at $0.65 \mathrm{~nm}$ or $1.12 \sim 1.18 \mathrm{~nm}$, offering an efficient dispersion medium to stabilize the graphene sheets. Although the pristine multilayer graphene sheets present similar stability in different glycerol/urea solvents, the U-type graphene experiences distinct levels of stabilization in solvents in the order of pure glycerol $>$ glycerol/urea(2/1) $>$ glycerol/urea(3/1) $>$ glycerol/urea(1/1), signifying that the shifted and exfoliated state of the graphene sheets plays an important role in the stabilization during liquid-phase exfoliation. Moreover, in the glycerol/urea binary solvents, the small urea molecules firstly diffuse into the graphene interlayer due to their strong $\pi-\pi$ interaction with graphene, acting as a "dispersion initiator". And then the glycerol molecules could have the chance to diffuse around or insert into the graphene interlayer to assist in stabilizing the graphene due to the hydrogen bonding between urea and glycerol. In this way, the glycerol helps to further increase the interlayer separation leading to a more stable dispersion, acting as a "dispersion co-stabilizer". The formation of the confined urea and glycerol solvents thus provides a stable molecular layer structure between the graphene interlayer, enabling to stabilize the exfoliated graphene sheets effectively. As such, the findings in this work are believed to provide the atomic/molecular scale understanding of the stability behavior of the graphene sheets in glycerol/urea binary solvents during liquid-phase exfoliation.

Keywords molecular dynamics; stability; graphene; glycerol; urea
\end{abstract}

*E-mail: libei@whut.edu.cn

Received October 13, 2020; published February 20, 2021.

Project supported by the National Natural Science Foundation of China (No. 51603160).

项目受国家自然科学基金青年基金(No. 51603160)资助. 


\section{1 引言}

单层石墨烯(graphene)是一种仅一个碳原子厚度的 二维纳米材料, 由碳原子以 $\mathrm{sp}^{2}$ 杂化轨道组成, 呈平面 正六边形的蜂窝结构 ${ }^{[1-3]}$. 石墨烯特殊的结构, 赋予其优 异的物理化学性能, 如杨氏模量将近 $1.0 \mathrm{TPa}^{[4-5]}$, 断裂 强度为 $42 \mathrm{~N} / \mathrm{m}^{[6]}$, 电子迁移率高达 $2 \times 10^{5} \mathrm{~cm}^{2} /(\mathrm{V} \bullet \mathrm{s})^{[7]}$, 热导率可达 $5.30 \times 10^{3} \mathrm{~W} /(\mathrm{m} \bullet \mathrm{K})^{[8]}$. 因此, 石墨烯在复合 材料 ${ }^{[9-12]}$ 、电子器件 ${ }^{[13-14]}$ 、能量存储 ${ }^{[15-17]}$ 、传感器 ${ }^{[18-20]}$ 及 生物医药 ${ }^{[21-24]}$ 等领域有着广泛的应用.

目前，石墨烯的制备方法已经由低效率的机械剥离 方法 ${ }^{[1,25]}$ 转向产量更大的外延生长法 ${ }^{[26-27]}$ 、化学气相沉 积法 ${ }^{[28-29]}$ 、氧化还原法 ${ }^{[30-31]}$ 和液相剥离法 ${ }^{[32-33]}$. 其中, 液相剥离法简单、高效且能够制备出高纯度、高质量的 石墨烯, 已成为制备石墨烯的重要方法. 液相剥离法的 基本原理 ${ }^{[34]}$ 是借助外力作用(如超声波、机械摚拌或者 热处理等)使得石墨层间距离增大, 层间范德华相互作 用减弱, 有利于剥离剂分子和溶剂分子等进入到石墨层 间, 对层层堆叠的石墨进行剥离和分散, 最后通过对石 墨烯剥离液进行离心抽滤和真空干燥等, 从而得到高质 量的石墨烯片. 因此, 寻找有利于剥离、绿色高效且易 去除的有机溶剂成为了研究液相剥离石墨烯的重点和 难点. Coleman ${ }^{[35-36]}$ 对石墨烯的液相剥离进行了深入地 理论分析, 发现有效剥离石墨烯的溶剂往往可以通过溶 剂的 Hildebrand 和 Hansen 溶剂化参数以及溶剂的表面 张力来挑选. Hernandez 等 ${ }^{[32-33]}$ 提出, 当溶剂与石墨烯表 面能接近时, 溶剂与石墨烯的混合熵更小, 对石墨的剥 离效果更明显, 因此一般所选溶剂的表面张力为 $40 \sim$ $50 \mathrm{~mJ} / \mathrm{m}^{2}$. 然而, 这些半经验理论仅提供了选择剥离剂 的方向, 并不能够为剥离和稳定石墨烯提供原子/分子 尺度的理解, 因此不能用于分子水平设计新型剥离剂和 稳定剂.

分子动力学 (molecular dynamics, 简称 MD)模拟方 法可以提供直观的原子/分子尺度信息, 能够描述石墨 烯剥离过程中微观结构的演变和石墨烯与溶剂之间的 相互作用等. 因此, MD 模拟是研究液相剥离石墨烯的 重要手段. Shih 等 ${ }^{[37]}$ 利用 MD 模拟方法研究了石墨烯在 5 种极性溶剂( $N$-甲基吡咯烷酮(NMP)、 $N, N$-二甲基甲酰 胺(DMF)、二甲基亚砜(DMSO)、 $\gamma$-羟基丁酸内酯(GBL) 和水)中的稳定性强弱, 平均力势表明溶剂对石墨烯的 稳定性关系为: $\mathrm{NMP} \approx \mathrm{DMSO}>\mathrm{DMF}>\mathrm{GBL}>\mathrm{H}_{2} \mathrm{O} . \mathrm{Fu}$ 等 ${ }^{[38]}$ 采用 MD 模拟方法研究了石墨烯在 NMP、DMSO 以及水等溶剂介质中的相互作用, 结果表明 NMP 和 DMSO 对石墨烯表面有较强的亲和力, 而石墨烯具有疏 水性. Yang 等 ${ }^{[39]}$ 总结了近年来利用 MD 模拟研究液相剥 离和稳定石墨烯的进展, 发现石墨烯与溶剂的界面相互 作用可用来表征石墨烯的稳定性, 其中包括石墨烯层间 的受限溶剂层产生的溶剂诱导力、带电石墨烯层间的静 电排斥力以及来自表面活性剂的诱导力. Xu 等 ${ }^{[40]}$ 使用
$\mathrm{MD}$ 模拟，探讨了在压缩二氧化碳、对二甲苯和萠基聚 环氧乙烯(Py-PEG)复合溶剂中石墨烯的稳定性机理. 研 究表明 Py-PEG 上的苯环与石墨烯的相互吸引作用和空 间位阻效应，能够有效防止石墨烯贴合.

近年来, 用于液相剥离石墨烯的溶剂(如: DMF、 NMP、GBL、DMSO 等)多属于有毒、高沸点且价格昂 贵的有机溶剂, 在制备石墨烯过程中可能会给人体带来 伤害 ${ }^{[41]}$, 而且剥离过程往往采用超声波处理, 导致石墨 烯尺寸减小、缺陷增多 [42], 并且所制备的石墨烯剥离液 难以长期稳定. 有研究表明 ${ }^{[43]}$, 将石墨分散于一定物质 的量比的甘油/尿素二元溶剂中, 进行 $8 \mathrm{~h} 、 150 \mathrm{r} / \mathrm{min}$ 的 机械搅拌处理, 再经过 $30 \mathrm{~min} 、 4000 \mathrm{r} / \mathrm{min}$ 离心搅拌, 即 可制备出尺寸大、缺陷少的天然石墨烯. 该方法剥离石 墨烯效率高达 $12.6 \mathrm{~g} / \mathrm{h}$, 剥离液能稳定保持长达 10 个月, 同时甘油/尿素属于环境友好型、价格低廉且易去除的有 机溶剂. 然而, 实验中难以实时观测石墨烯在溶剂体系 中的结构演变, 石墨烯与甘油/尿素溶剂之间的相互作 用以及石墨烯稳定的微观机理尚不明确. 因此, 本工作 通过 MD 模拟石墨烯在甘油/尿素二元溶剂体系中的结 构变化, 讨论石墨烯在不同配比溶剂中的稳定性, 并选 用纯甘油溶剂作为比对模型, 进一步分析溶剂对石墨烯 的稳定性机理.

\section{2 分子动力学计算}

\section{1 模型构建}

\subsection{1 多层石墨烯/溶剂模型}

本工作通过改变石墨烯层间距，发现层间距不小于 $0.6 \mathrm{~nm}$ 时, 溶剂能够进入石墨烯层间, 形成溶剂分子层, 从而有助于石墨烯在剥离后稳定存在. 另一方面, 分子 间范德华和静电作用的截断半径为 $1.2 \mathrm{~nm}$, 即石墨烯层 间距大于 $1.2 \mathrm{~nm}$ 时, 石墨烯层间相互作用消失, 可认为 完全剥离. 因此, 为了探讨不同层间距下石墨烯在溶剂 中的稳定性，本工作构建了石墨烯初始层间距分别为 $0.6 \mathrm{~nm}$ (部分剥离)和 $1.2 \mathrm{~nm}$ (完全剥离)的多层石墨烯模 型(如图 1(a)所示), 将其放置于 $6 \mathrm{~nm} \times 6 \mathrm{~nm} \times 6 \mathrm{~nm}$ 的模 拟盒子中, 并根据不同甘油/尿素物质的量比填充溶剂 分子, 具体参数如表 1 所示. 特别地, 为了考察溶剂分 子与石墨烯间的相互作用，石墨烯层间在初始时刻未填 充溶剂分子.

\subsubsection{U 型石, 墨烯/溶剂模型}

考虑到液相剥离中, 石墨烯会呈现出部分㲐羾曲变形 且未完全剥离的状态, 即层间贴合面积较小, 结构严重 变形, 因此构建尺寸为 $4.912 \mathrm{~nm} \times 4.963 \mathrm{~nm}$ 的 U 型石墨 烯, 如图 1(b)所示. 已测试出在甘油/尿素溶剂(物质的 量比为 2/1)中, 同样尺寸的双层和多层 U 型石墨烯的稳 定行为相同. 因此, 为提高计算效率, 本工作采用双层 $\mathrm{U}$ 型石墨烯来研究甘油/尿素二元溶剂对石墨烯的稳定 性影响, 具体参数如表 2 所示. 
(a)

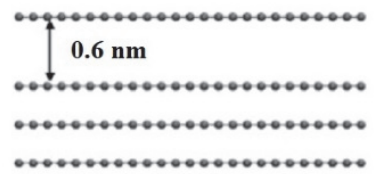

(b)

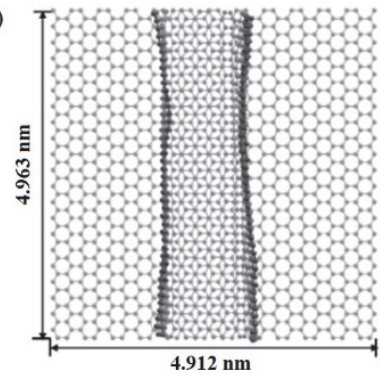

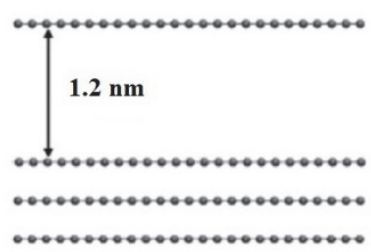

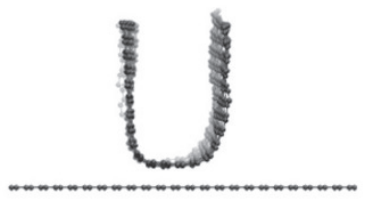

图 1 (a) 多层石墨烯(初始层间距分为 $0.6 \mathrm{~nm}$ 和 $1.2 \mathrm{~nm}$ )和(b) $\mathrm{U}$ 型石 墨烯原子模型

Figure 1 Atomic models of the (a) pristine multilayer graphene with the initial interlayer spacing of $0.6 \mathrm{~nm}$ and $1.2 \mathrm{~nm}$, and (b) U-type graphene

\section{2 模拟方法}

本工作采用 GROMACS 软件, 并使用 GAFF 力场 (该力场被广泛用于研究石墨烯在有机、无机溶剂体系 中的结构与性能等 ${ }^{[4-47]}$ )完成所有模拟计算. 石墨烯、甘 油和尿素分子的 $\mathrm{GAFF}$ 力场参数均由 $\mathrm{ACPYPE}{ }^{[48]}$ 软件 生成, 并将石墨烯视为不带电荷的Lennard-Jones 势粒 子 ${ }^{[49]}$. 本工作选取的积分时间步长为 $2 \mathrm{fs}$, 并在 $x 、 y$ 和 $z$ 方向上均采用周期性边界条件. 范德华和静电相互作 用截断半径为 $1.2 \mathrm{~nm}$, 并使用 LINCS $^{[50-51]}$ 算法约束分子 键长, $P M E^{[52-53]}$ 求和方法计算长程静电力.

模拟过程分为以下三步:

(1)对初始模型进行能量最小化. 采用最速下降 法 ${ }^{[54]}$, 体系能量收玫标准为 $10.0 \mathrm{~kJ} /(\mathrm{mol} \bullet \mathrm{nm})$.

(2)固定石墨烯, 平衡溶剂体系. 选用 NPT 系综, 采

用 Berendsen ${ }^{[5]}$ 方法控压, 耦合压力为 $0.1 \mathrm{MPa}$, V-rescale ${ }^{[56]}$ 方法控温，耦合温度为 $298.15 \mathrm{~K}$, 模拟时间 为 1 ns. 再采用 Parrinello-Rahman ${ }^{[57]}$ 方法控压, NoseHoover ${ }^{[58]}$ 方法控温，模拟时间为 2 ns.

(3)解除顶层石墨烯的约束, 固定下层石墨烯, 平衡 顶层石墨烯. 选用 NVT 系综, 采用 Nose-Hoover 方法控 温, 耦合温度为 $298.15 \mathrm{~K}$, 模拟时间为 $10 \mathrm{~ns}$ (多层石墨 烯)或 $5 \mathrm{~ns}$ (U 型石墨烯).

本工作通过对比石墨层间距(或单层石墨烯厚度)和 甘油、尿素的密度值来验证 GAFF 力场参数的准确性. 经计算, 石墨层间距为 $0.337 \mathrm{~nm}$, 实验值为 $0.335 \mathrm{~nm}^{[59]}$, 误差仅为 $0.597 \%$. 甘油和尿素的密度模拟值分别为

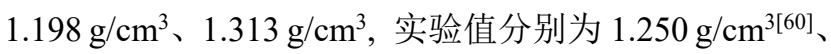
$1.335 \mathrm{~g} / \mathrm{cm}^{3[61]}$, 误差分别为 $4.160 \%$ 和 $1.648 \%$. 模拟值与 实验值相比, 误差均小于 $5 \%$. 由此可知, GAFF 力场能 够合理、有效地描述石墨烯、甘油和尿素分子.

\section{3 结果与讨论}

\section{1 多层石墨烯的稳定行为}

\subsection{1 石墨烯微观形貌演变}

图 2 分别展示了初始层间距为 $0.6 \mathrm{~nm}$ 和 $1.2 \mathrm{~nm}$ 时, 石墨烯在纯甘油和不同物质的量比的甘油/尿素溶剂中 的微观形貌演变. 可以发现，当初始层间距为 $0.6 \mathrm{~nm}$ 时, NPT 系综下, 尿素分子能够进入石墨烯层间, 并形成单 层分子层, 而甘油分子体积相对较大, 未能进入石墨烯 层间. 接下来, NVT 系综下, 在甘油/尿素溶剂中, 顶层 石墨烯因约束解除, 结构出现褶皱、趐曲和侧移变化, 且石墨烯层间边缘处进入少量甘油分子. 而在纯甘油溶 剂中，石墨烯发生完全贴合，未发生明显的尧曲和偏移. 当初始层间距增加至 $1.2 \mathrm{~nm}$ 时, 在 NPT 系综下, 对于 各溶剂体系, 已剥离的石墨烯层间均可形成两层溶剂分

表 1 不同甘油/尿素物质的量比下的多层石墨烯/溶剂体系模拟参数

Table 1 Parameters for pristine multilayer graphene/solvent systems with different glycerol/urea concentrations

\begin{tabular}{|c|c|c|c|c|c|}
\hline 石墨烯尺寸 $/ \mathrm{nm}^{2}$ & 初始层间距/nm & 溶剂 & 甘油分子数 & 尿素分子数 & 模拟盒子初始尺寸 $/ \mathrm{nm}^{3}$ \\
\hline \multirow{8}{*}{$2.944 \times 2.996$} & \multirow{4}{*}{0.6} & 纯甘油 & 1432 & 0 & \multirow{8}{*}{$6 \times 6 \times 6$} \\
\hline & & 甘油/尿素(3/1) & 1251 & 417 & \\
\hline & & 甘油/尿素(2/1) & 1142 & 571 & \\
\hline & & 甘油/尿素(1/1) & 935 & 935 & \\
\hline & \multirow{4}{*}{1.2} & 纯甘油 & 1442 & 0 & \\
\hline & & 甘油/尿素(3/1) & 1257 & 419 & \\
\hline & & 甘油/尿素(2/1) & 1154 & 577 & \\
\hline & & 甘油/尿素(1/1) & 939 & 939 & \\
\hline
\end{tabular}

表 2 不同甘油/尿素物质的量比下的 U 型石墨烯/溶剂体系模拟参数

Table 2 Parameters for U-type graphene/solvent systems with different glycerol/urea concentrations

\begin{tabular}{ccccc}
\hline 石墨烯尺寸 $/ \mathrm{nm}^{2}$ & 溶剂体系 & 甘油分子数 & 尿素分子数 & 模拟盒子初始尺寸 $/ \mathrm{nm}^{3}$ \\
\hline \multirow{4}{*}{$4.912 \times 4.963$} & 纯甘油 & 4227 & 0 & $9 \times 9 \times 9$ \\
& 甘油/尿素(3/1) & 3471 & 1157 & \\
& 甘油/尿素 $(2 / 1)$ & 3188 & 1594 & \\
& 甘油/尿素 $(1 / 1)$ & 2558 & 2558 & \\
\hline
\end{tabular}


(I) $0.6 \mathrm{~nm}$

(a)

(b)
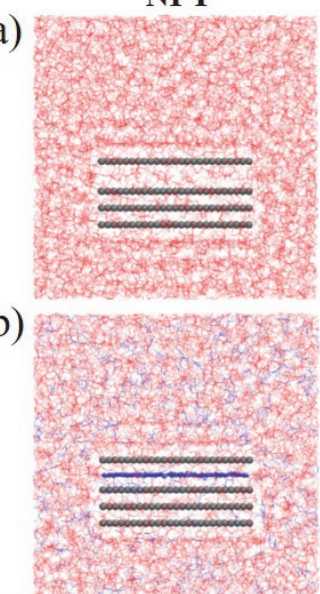

(c)

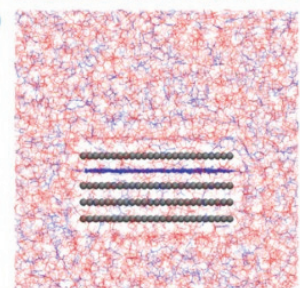

(d)

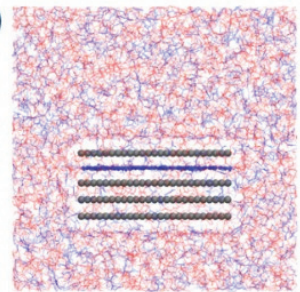

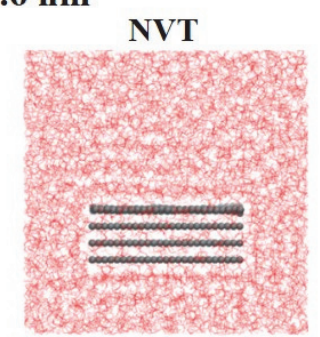
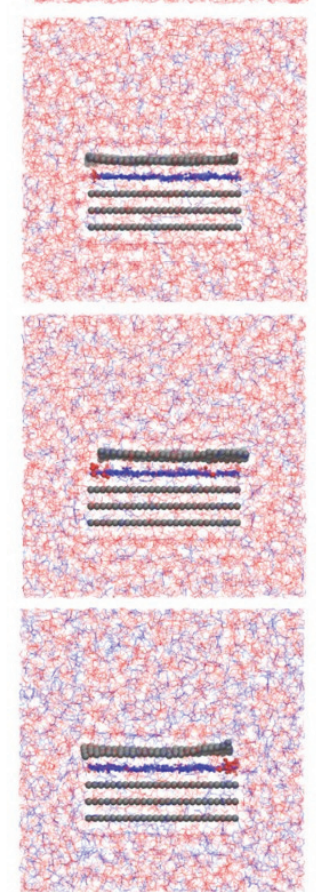

(II) $1.2 \mathrm{~nm}$

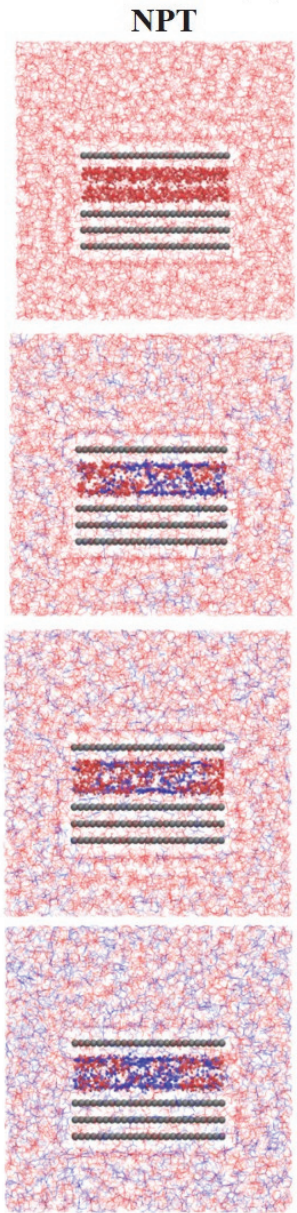

NVT
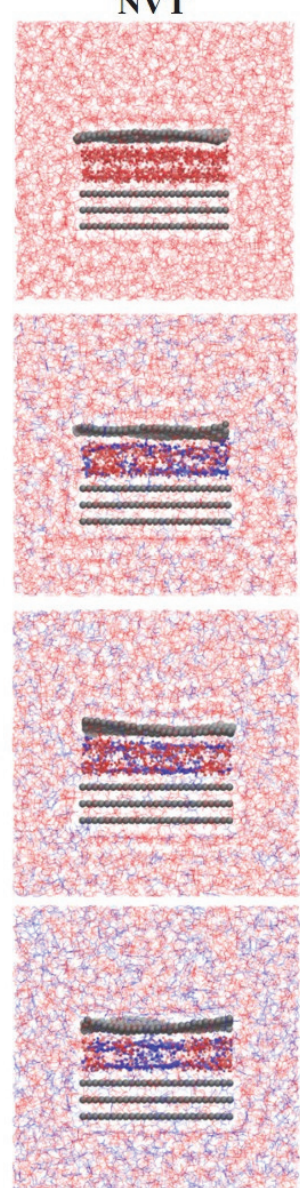

图 2 初始层间距分别为(I) $0.6 \mathrm{~nm}$ 和(II) $1.2 \mathrm{~nm}$ 时, 多层石墨烯在(a) 纯甘油,(b) 甘油/尿素(3/1), (c) 甘油/尿素(2/1)和(d) 甘油/尿素(1/1)溶剂体系 中的 NPT 和 NVT 系综下的最终结构. 其中灰色表示石墨烯片层, 红色表示甘油分子, 蓝色表示尿素分子, 高亮加粗部分为层间受限溶剂分子

Figure 2 The final structure of the pristine multilayer graphene in solvents of (a) pure glycerol, (b) glycerol/urea(3/1), (c) glycerol/urea(2/1) and (d) glycerol/urea(1/1) with the initial interlayer spacing of (I) $0.6 \mathrm{~nm}$ and (II) $1.2 \mathrm{~nm}$ using NPT and NVT ensemble, respectively. The graphene sheets are colored as gray, and the glycerol and urea molecules are red and blue. In particular, the solvent molecules between the top and second graphene sheets are highlighted

子层, 但甘油、尿素分子分布并不均匀. 在 NVT 系综下, 石墨烯层间溶剂分子层相对稳定，层间距变化不大，但 石墨烯故曲结构较显著.

\subsection{2 石墨烯结构分析}

(1)均方根偏差

为了描述石墨烯微观结构的变化程度, 反映各溶剂 体系对石墨烯的稳定性差异, 分析了 NVT 系综下顶层 石墨烯构型的均方根偏差 $(\mathrm{RMSD})^{[62]}$, 具体计算如下:

$$
\operatorname{RMSD}=\sqrt{\frac{\sum_{i=0}^{N}\left[m_{i} \times\left(X_{i}^{0}-X_{i}^{\prime}\right)^{2}\right]}{M}}
$$

其中 $N 、 M 、 m_{i} 、 X_{i}^{0} 、 X_{i}^{\prime}$ 分别表示原子总数、原子总质 量、第 $i$ 个原子的质量、初始结构和当前结构中第 $i$ 个 原子的坐标矢量. RMSD 是一个相对值, 值越大, 说明 顶层石墨烯与初始结构差异越大.
由图 3(a)可知, 当初始层间距为 $0.6 \mathrm{~nm}$ 时, 石墨烯 在纯甘油溶剂中的 RMSD 值迅速增加, 并稳定在 0.275 $\mathrm{nm}$ 附近. 这是因为石墨烯未完全剥离, 且层间无溶剂 分子, 已部分剥离的石墨烯因层间 $\pi-\pi$ 相互作用能快速 发生完全贴合，同时石墨烯表面出现褶皱. 随后，多层 石墨烯结构稳定, RMSD 值亦保持稳定. 而对于甘油/尿 素溶剂体系, 虽然单层受限分子层能有效阻止石墨烯贴 合, 但在约束解除后石墨烯表面仍将快速出现褶皱, 引 起 RMSD 值迅速增加. 接下来在不同溶剂的作用下, 石 墨烯结构进一步发生变化, 但 RMSD 值均较小. 虽然在 物质的量比为 $2 / 1$ 时, RMSD 值相对较大, 但仍反映了 甘油/尿素溶剂在不同物质的量比下对石墨烯均具有较 强的稳定作用. 而当石墨烯处于完全剥离状态时(初始 层间距为 $1.2 \mathrm{~nm}$ ), 各溶剂体系中石墨烯的 RMSD 值和 变化趋势均相似(图 3(b)), 说明各溶剂体系对已剥离石 墨烯的稳定性差异不显著. 

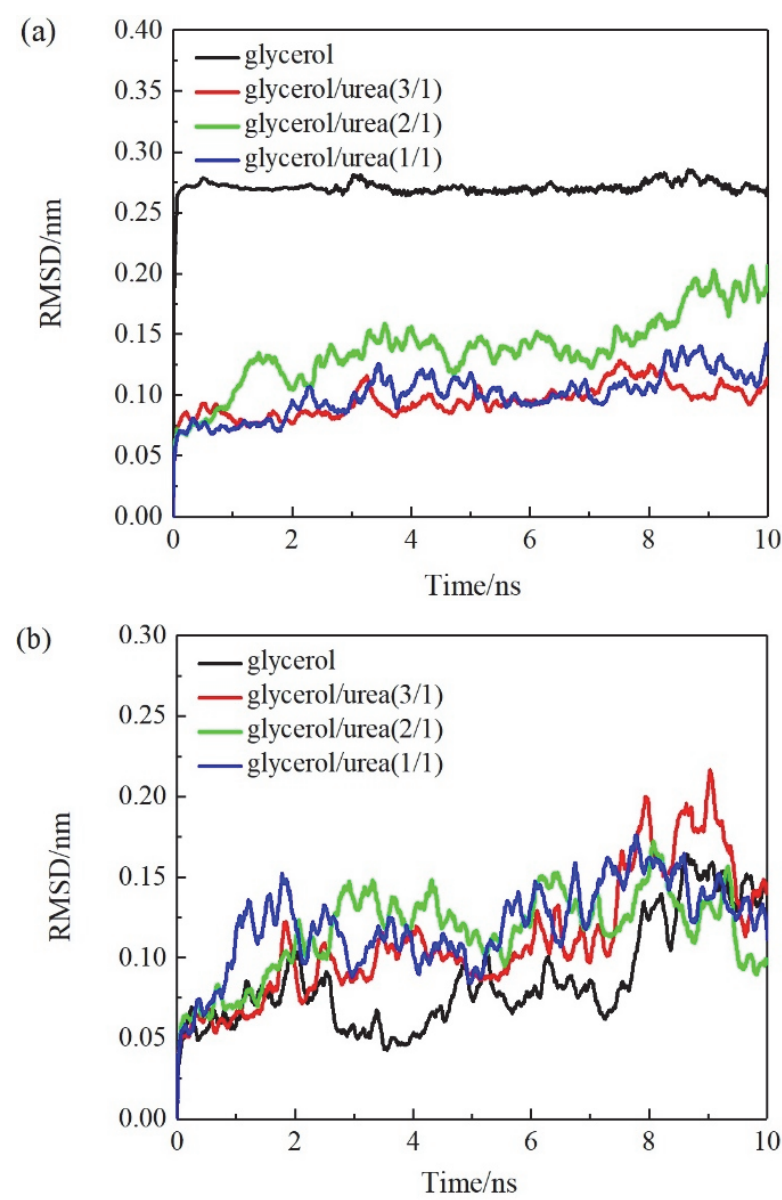

图 3 初始层间距为(a) $0.6 \mathrm{~nm}$ 和(b) $1.2 \mathrm{~nm}$ 时, 顶层石墨烯在 NVT 系 综下的 RMSD 变化曲线

Figure 3 Evolution of the RMSD of the top graphene sheet under NVT with the initial interlayer spacing of (a) $0.6 \mathrm{~nm}$ and (b) $1.2 \mathrm{~nm}$

\section{(2)层间距离}

图 4 计算了石墨烯层间距离变化, 进一步说明溶剂 体系对石墨烯稳定性的影响. NPT 系综下, 石墨烯整体 固定不动, 石墨烯层间距保持不变. NVT 系综下, 顶层 石墨烯约束解除, 其在溶剂及底部石墨烯片层的共同作 用下发生运动. 由图 4(a)可知, 在纯甘油溶剂中, 石墨 烯层间距离迅速由 $0.6 \mathrm{~nm}$ 减小为约 $0.336 \mathrm{~nm}$, 与石墨层 间距(或石墨烯厚度)相一致, 说明石墨烯快速发生贴合. 在甘油/尿素溶剂中, 石墨烯层间距离则因表面褶皱的 出现而迅速增大, 并在平衡过程中层间距略微增大至约 $0.65 \mathrm{~nm}$, 这与石墨烯结构演变一致, 说明甘油/尿素溶 剂对石墨烯具有一定的稳定作用. 图 4(b)显示, 当石墨 烯初始层间距增加至 $1.2 \mathrm{~nm}$ 时, 各溶剂体系下的层间距 虽略有减小 $(1.12 \sim 1.18 \mathrm{~nm})$, 但均未贴合, 说明甘油和 甘油/尿素二元溶剂均对石墨烯具有稳定作用. 但由于 甘油分子体积较大, 提供的空间位阻更大, 因此在纯甘 油中石墨烯层间距更大.

(3)侧移距离

为了反映石墨烯在不同溶剂中剥离趋势的强弱, 可 (a)

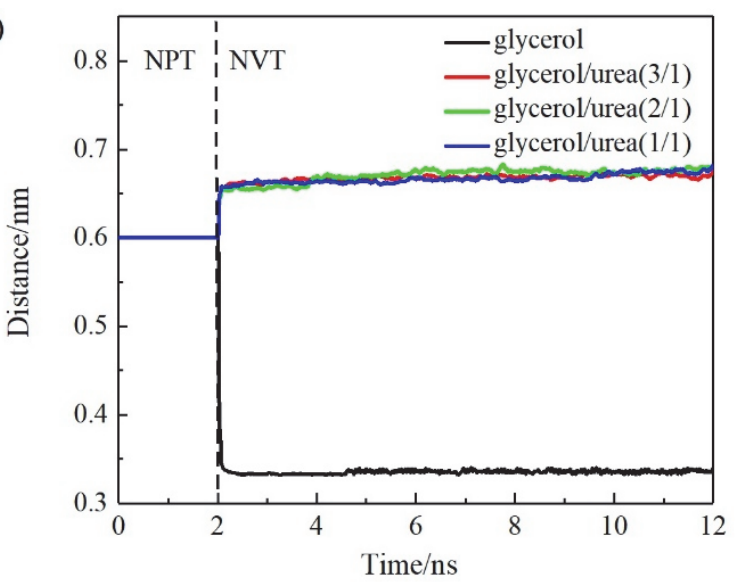

(b)

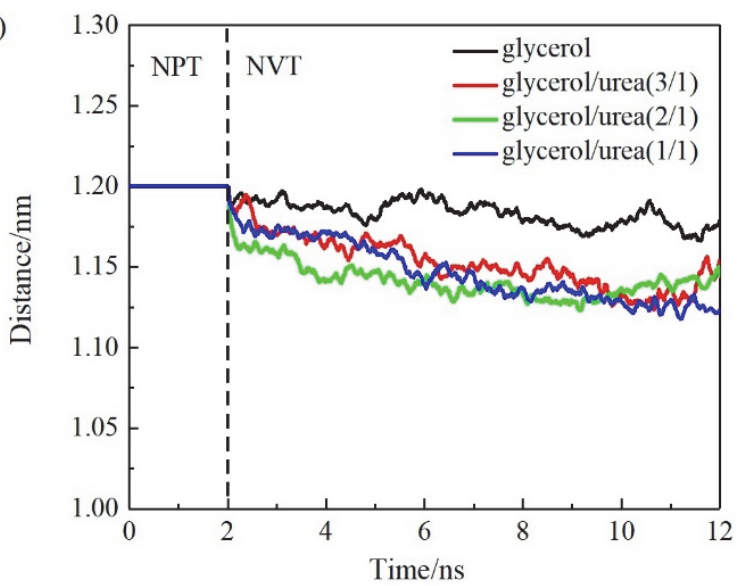

图 4 初始层间距为(a) $0.6 \mathrm{~nm}$ 和(b) $1.2 \mathrm{~nm}$ 时, 石墨烯层间距离变化 曲线

Figure 4 Evolution of the interlayer distance between the top and second layers of the graphene with the initial spacing of (a) $0.6 \mathrm{~nm}$ and (b) $1.2 \mathrm{~nm}$

考察 NVT 系综下顶层石墨烯质心发生侧移的距离. 在 此，将石墨烯质心与其初始时刻的质心在水平方向上的 距离偏差定义为侧移距离.

图 5 显示了石墨烯在初始层间距为 $0.6 \mathrm{~nm}$ 和 1.2 $\mathrm{nm}$ 时的侧移距离变化曲线. 由图 5(a)可知, 在物质的量 比为 $2 / 1$ 时, 石墨烯的侧移距离最大, 且具有增长趋势, 说明该物质的量比下的甘油/尿素二元溶剂对石墨烯具 有较强的稳定和剥离作用, 同时这也解释了该体系下石 墨烯的 RMSD 值较大的原因. 而另外三种溶剂体系的 偏移距离变化较小, 对石墨烯主要表现为稳定作用. 而 当石墨烯已完全剥离时(图 5(b)), 各溶剂体系中, 石墨 烯的侧移距离虽有所增大，但剥离作用不明显，对石墨 烯主要表现均为稳定作用.

\subsection{3 溶剂分布}

为了揭示各溶剂组分对石墨烯稳定性的影响, 计算 了石墨烯层间溶剂分子数目变化, 如图 6 所示.

当初始层间距为 $0.6 \mathrm{~nm}$ 时, NPT 系综下, 尿素分子 因其体积较小, 且与石墨烯具有较强的相互吸引作用, 能够迅速进入狭窄的石墨烯层间. 当尿素分子充满石墨 

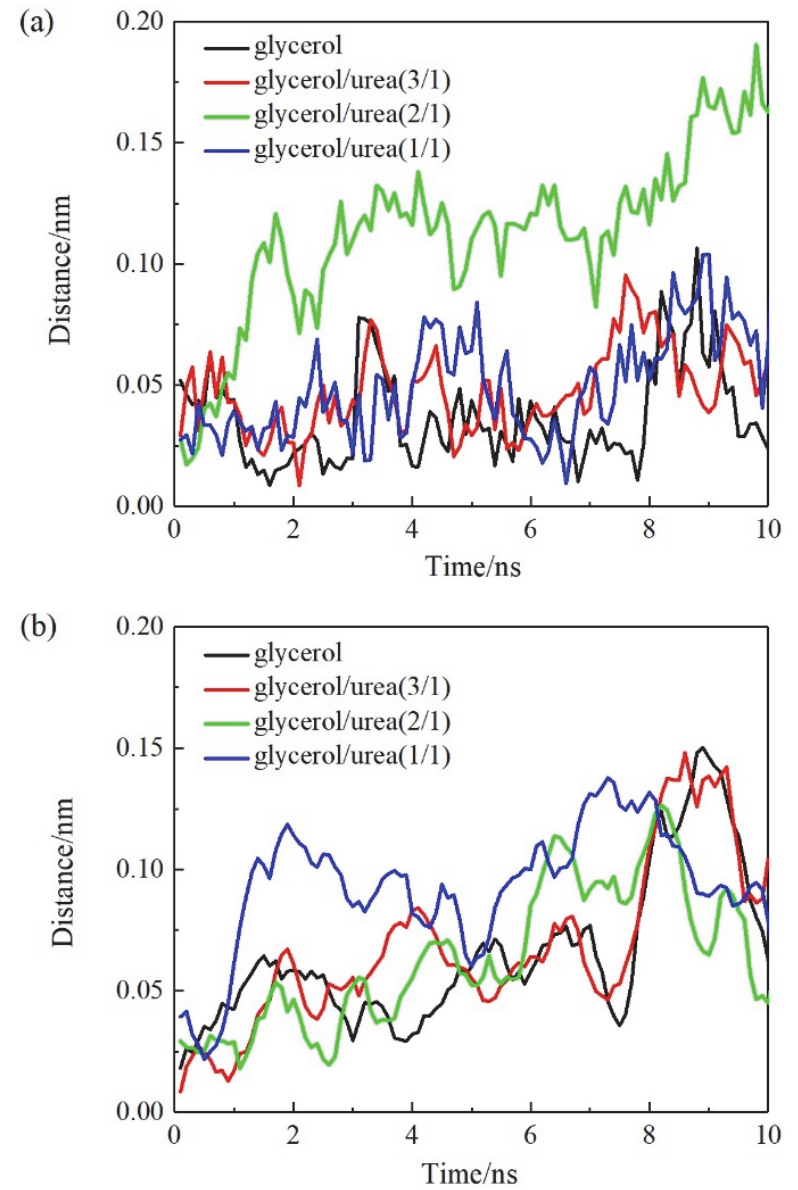

图 5 初始层间距为(a) $0.6 \mathrm{~nm}$ 和(b) $1.2 \mathrm{~nm}$ 时, 顶层石墨烯在 NVT 系 综下的侧移距离曲线

Figure 5 Evolution of the lateral shift distance of the top graphene sheet under NVT with the initial interlayer spacing of (a) $0.6 \mathrm{~nm}$ and (b) $1.2 \mathrm{~nm}$
烯层间后，尿素分子数目基本不变，如图 6(a) 6(c) 所 示. 而在 NVT 系综，石墨烯层间甘油分子开始略有增 加. 这是因为层间的尿素分子处于挤压状态 ${ }^{[37]}$ ，当解除 顶层石墨烯约束后，尿素分子能与石墨烯形成较稳定的 $\pi-\pi$ 共轭作用，分子结构得以舒展，增加了石墨烯层间 距. 同时, 甘油与尿素能够形成氢键, 从而在尿素的牵 引作用下进入石墨烯层间. 图 6(d) 6(g)显示, 当石墨 烯层间距较大 $(1.2 \mathrm{~nm})$ 时, 在 NPT 系综, 甘油、尿素分 子均能够进入石墨烯层间，并形成双层溶剂分子层(见 图 2). 注意到, 在甘油/尿素二元溶剂中，尿素分子数大 于甘油分子数，因此石墨烯层间尿素浓度均大于层外. 这是因为尿素与石墨烯间较强的相互吸引作用，尿素能 够更快、更多地进入石墨烯层间，并分布在石墨烯中心 区域; 而甘油通过氢键作用牵引至石墨烯层间, 主要分 布在石墨烯边缘区域. 在NVT系综, 石墨烯层间溶剂分 子数目变化不大, 这是因为石墨烯层间距减小后(图 4(b)), 层间溶剂运动能力变差. 但受限溶剂分子能够阻 止石墨烯聚集, 为石墨烯分散和稳定提供了保障. 这也 说明石墨烯层间能否形成稳定受限分子层是石墨烯在 剥离液中保持稳定的关键.

\section{$3.2 \cup$ 型石墨烯的稳定行为}

\subsection{1 石墨烯微观形貌演变}

由图 7 可知, $\mathrm{U}$ 型石墨烯在各溶剂体系下均发生了 结构改变, 呈现出一定程度的贴合和侧移, 说明纯甘 油、甘油/尿素二元溶剂对 U 型石墨烯具有一定的稳定 性. 特别地, 在纯甘油中, $U$ 型石墨烯稳定性最好, 而在 甘油/尿素物质的量比为 $1 / 1$ 时稳定性最差.
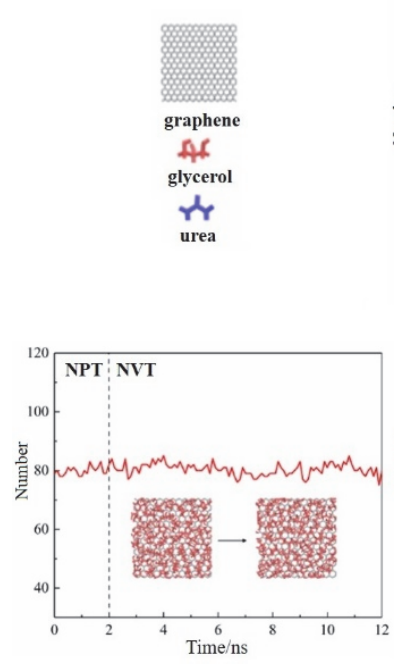

(d)

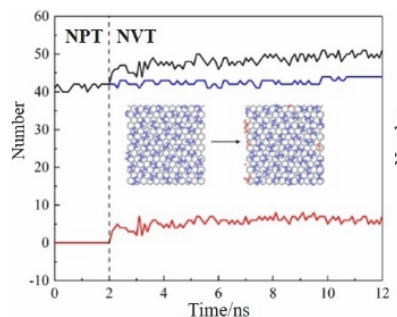

(a)

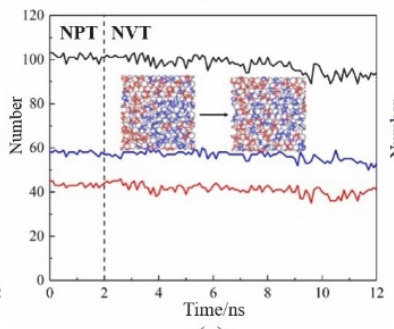

(e)

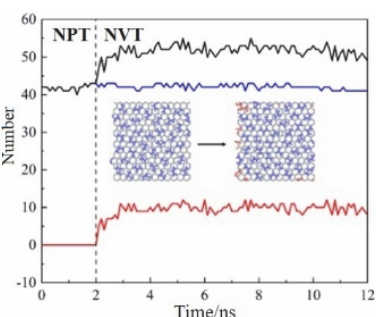

(b)

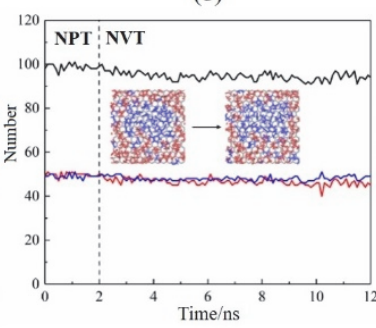

(f)

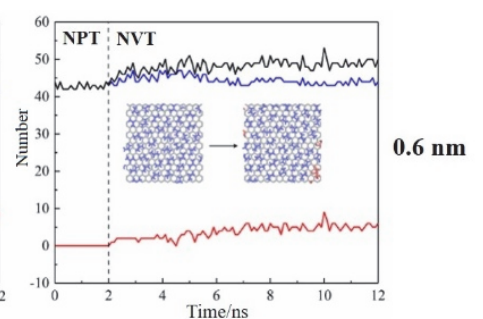

(c)

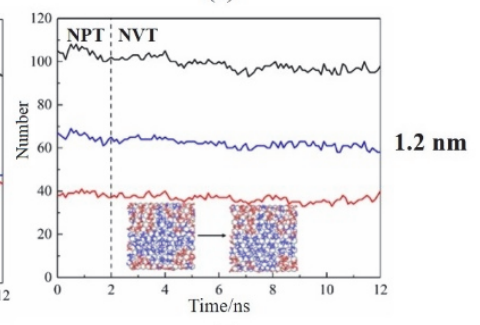

(g)

图 6 初始层间距离为 $0.6 \mathrm{~nm}$ 时, (a) 甘油/尿素(3/1), (b) 甘油/尿素(2/1)和(c) 甘油/尿素(1/1)溶剂体系中的甘油(红线)、尿素(蓝线)和所有溶剂(黑 线)的分子数目变化曲线. 初始层间距离为 $1.2 \mathrm{~nm}$ 时, (d) 纯甘油, (e) 甘油/尿素(3/1), (f) 甘油/尿素(2/1)和(g) 甘油/尿素(1/1)溶剂体系中的甘油(红 线)、尿素(蓝线)和所有溶剂(黑线)的分子数目变化曲线, 其中插图分别为 NPT 和 NVT 系综下最终层间溶剂分布

Figure 6 Evolution of the number of glycerol (red line), urea (blue line) and all organic (black line) molecules in solvents of (a) glycerol/urea(3/1), (b) glycerol/urea(2/1) and (c) glycerol/urea(1/1) with the initial interlayer spacing of $0.6 \mathrm{~nm}$, and those in (d) pure glycerol, (e) glycerol/urea(3/1), (f) glycerol/urea(2/1) and $(\mathrm{g})$ glycerol/urea(1/1) with the initial interlayer spacing of $1.2 \mathrm{~nm}$ under NPT and NVT ensemble, respectively. The insets indicate the final distribution of the constrained solvent molecules 
NPT

(a)

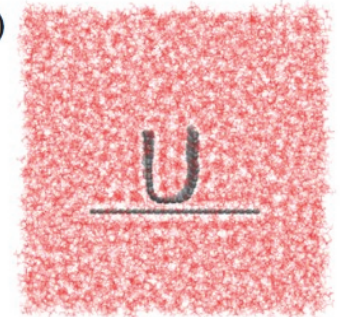

(c)

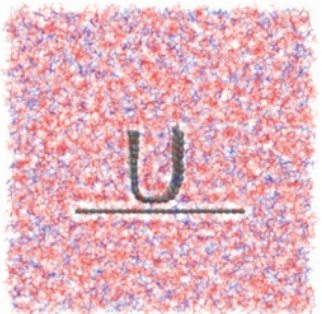

NVT
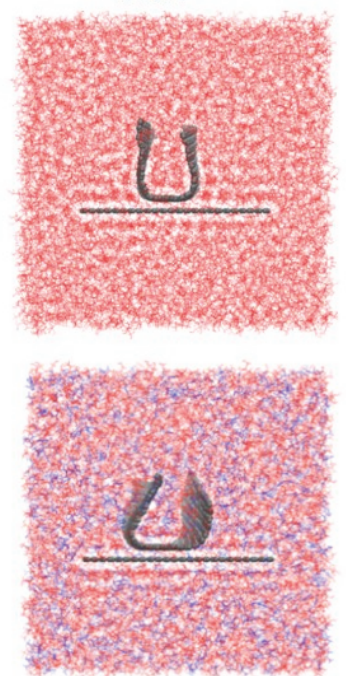

NPT

(b)

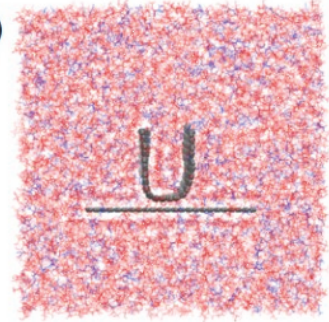

(d)

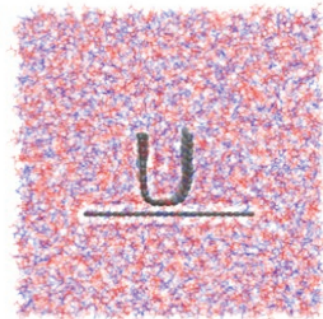

NVT
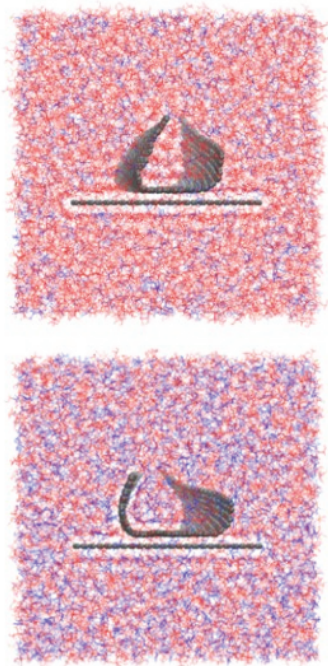

图 7 U 型石墨烯在 NPT 和 NVT 系综下的最终结构图: (a) 纯甘油, (b) 甘油/尿素(3/1), (c) 甘油/尿素(2/1)和(d) 甘油/尿素(1/1)溶剂体系

Figure 7 The final structure of U-type graphene sheets in solvents of (a) pure glycerol, (b) glycerol/urea(3/1), (c) glycerol/urea(2/1), and (d) glycerol/urea(1/1) under NPT and NVT ensemble, respectively

\section{2 .2 石墨烯结构分析}

(1)均方根偏差

图 8 展示了各溶剂体系下, U 型石墨烯的 RMSD 值 随时间的变化曲线. 可以发现, RMSD 值因约束解除、 出现褶皱而迅速增加. 并在 $1.2 \mathrm{~ns}$ 之前, 四条曲线几乎 重合, 这是因为初始 $U$ 型石墨烯的键、角和二面角严重 扭曲, 结构极不稳定, 因此在平衡模拟初期, 石墨烯在 各溶剂中会发生类似的伸展. 随后, 在纯甘油体系中, RMSD 值变化较小, 并趋于稳定, 说明了甘油对已弯曲 变形的石墨烯具有较强的稳定作用. 而对于甘油/尿素 二元溶剂体系, RMSD 值继续增加, 结构仍在变化, 但 RMSD 值仍小于 $0.4 \mathrm{~nm}$, 说明甘油/尿素溶剂对石墨烯 也具有一定的稳定作用. 比较各溶剂体系中 RMSD 值 大小, 可以判定各溶剂对石墨烯的稳定能力为: 纯甘油 $>$ 甘油/尿素(2/1) > 甘油/尿素(3/1) > 甘油/尿素(1/1).

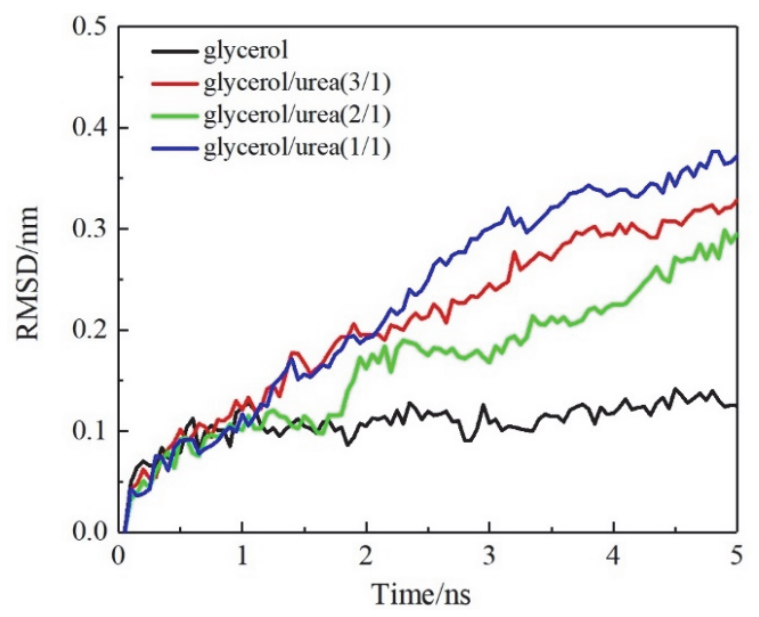

图 $8 \mathrm{U}$ 型石墨烯在 NVT 系综下的 RMSD 值变化曲线

Figure 8 Evolution of the RMSD of the U-type graphene under NVT

\section{(2)贴合面积}

由于热力学稳定下的石墨烯会发生一定的褶皱, 石 墨烯的层间距往往会大于 $0.335 \mathrm{~nm}^{[63]}$, 本工作定义石墨 烯层间距小于 $0.4 \mathrm{~nm}$ 时即为贴合. 因此, $\mathrm{U}$ 型石墨烯初 始贴合面积约占总面积的 $15 \%$.

为了直观且定量地描述平衡过程中, $\mathrm{U}$ 型石墨烯在 各溶剂体系下的贴合面积变化，计算了其贴合面积百分 比随时间变化的曲线, 如图 9 所示. 可以发现, 在各溶 剂体系中, $\mathrm{U}$ 型石墨烯不能完全保持其初始状态，均发 生一定程度的贴合，且贴合面积百分比顺序与 RMSD 值变化一致. 从贴合面积的增加趋势可知, 在纯甘油体 系中，贴合面积基本保持在 $21 \%$ 左右，说明甘油有利于 保持石墨烯的初始结构. 加入尿素后, 石墨烯的贴合面 积有所增大, 但在甘油/尿素物质的量比为 $2 / 1$ 溶剂体系 中的贴合面积最小，相对稳定. 计算各溶剂体系中 U 型 石墨烯的最终贴合面积占比分别为 $23 \% 、 31 \% 、 27 \%$ 、 $37 \%$, 进一步说明了 $U$ 型石墨烯在各溶剂体系中的稳定 性为: 纯甘油 $>$ 甘油/尿素(2/1) > 甘油/尿素(3/1) > 甘油/ 尿素(1/1).

\section{(3)侧移距离}

图 10 显示了各溶剂体系下 $\mathrm{U}$ 型石墨烯的侧移距离 随时间变化曲线. 在甘油/尿素物质的量比为 $2 / 1$ 溶剂体 系中, 石墨烯的侧移距离较大且呈增加趋势, 说明石墨 烯在该配比下剥离趋势最大, 且侧移距离是影响该体系 RMSD 变化的主要原因. 而对于纯甘油、甘油尿素物质 的量比为 $3 / 1$ 和 $1 / 1$ 溶剂体系, 侧移距离变化均较小且 稳定, 说明侧移距离对其 RMSD 值变化贡献相似且较 小. 此外, 根据侧移距离大小, 可进一步反映出各溶剂 体系对石墨烯的剥离能力顺序为: 甘油/尿素 $(2 / 1)>$ 甘 油/尿素(3/1) > 甘油/尿素(1/1) $>$ 纯甘油, 这与实验结 
果 ${ }^{[43]}$ 和计算模拟 ${ }^{[64]}$ 相吻合.

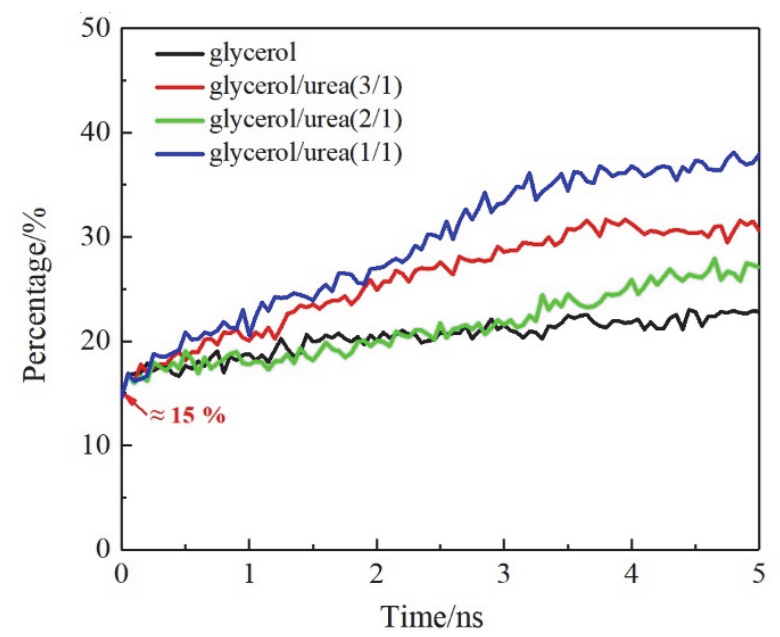

图 $9 \mathrm{U}$ 型石墨烯在 NVT 系综下的贴合面积百分比变化曲线

Figure 9 Evolution of the percentage of the contact area of the U-type graphene sheet under NVT

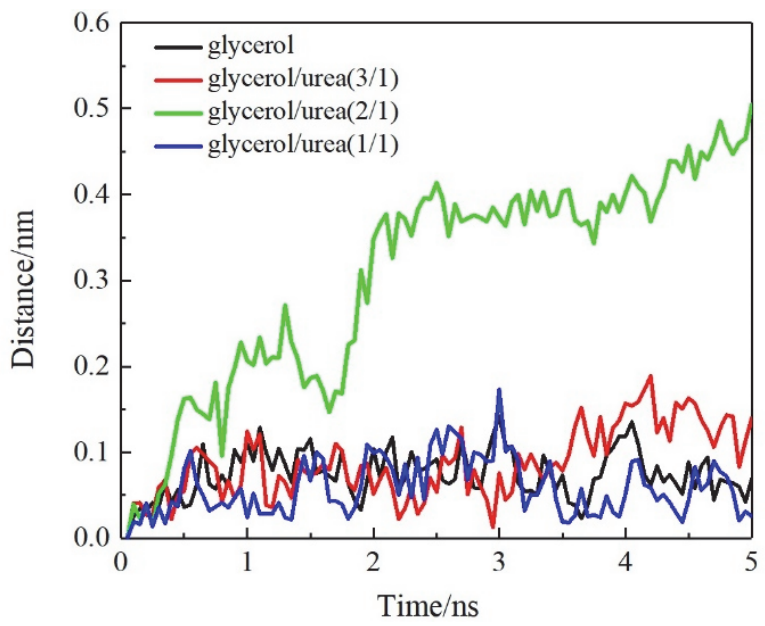

图 $10 \mathrm{U}$ 型石墨烯在 NVT 系综下的侧移距离变化曲线

Figure 10 The evolution of the lateral shift distance of the U-type graphene sheet under NVT

\section{4 结论}

本工作通过 MD 模拟, 研究了多层石墨烯和 U 型石 墨烯在甘油/尿素溶剂体系中的微观形貌和结构变化, 揭示了溶剂体系对石墨烯的稳定性影响及其机理. 具体 总结如下:

(1)通过分析石墨烯微观形貌, 发现初始层间距为 $0.6 \mathrm{~nm}$ 时, 多层石墨烯在纯甘油体系中发生贴合, 而在 甘油/尿素二元溶剂中均能稳定存在. 当初始层间距为 $1.2 \mathrm{~nm}$ 时, 石墨烯能够在各溶剂体系下稳定分散, 并无 显著差别. 对于 $U$ 型石墨烯, 在各溶剂体系中, 石墨烯 发生贴合和侧移均不同，表现出明显的稳定性差异.

(2)通过分析石墨烯结构变化, 发现多层石墨烯在 各溶剂体系中稳定性差异较小. 而 U 型石墨烯在各溶剂 体系中的稳定性顺序为: 纯甘油 $>$ 甘油/尿素(2/1) $>$ 甘
油/尿素(3/1)>甘油/尿素(1/1). 特别地, 由石墨烯侧移 距离可知, 石墨烯在溶剂中的稳定性与石墨烯的剥离状 态有关. 对于多层石墨烯, 各溶剂体系主要表现为稳定 作用. 而对于 $U$ 型石墨烯，因其未完全剥离，各溶剂体 系对石墨烯还表现出明显的剥离作用.

(3)通过分析溶剂分布, 发现当初始层间距为 0.6 $\mathrm{nm}$ 时，仅尿素分子能够进入石墨烯层间，增加石墨烯 层间距. 而甘油能够通过与尿素的氢键作用进入石墨烯 层间，进一步增大石墨烯层间距. 当初始层间距为 1.2 $\mathrm{nm}$ 时，甘油、尿素均能进入石墨烯层间，形成双层分子 层结构，阻止石墨烯聚集. 因此，尿素能在石墨烯层间 形成稳定的 $\pi-\pi$ 共轭结构, 为石墨烯在甘油/尿素二元溶 剂中的稳定提供了可行性. 受限溶剂分子与石墨烯存在 排斥作用，为石墨烯在甘油/尿素二元溶剂中长期稳定 提供了保障.

\section{References}

[1] Novoselov, K. S.; Geim, A. K.; Morozov, S. V.; Jiang, D.; Zhang, Y.; Dubonos, S. V.; Grigorieva, I. V.; Firsov, A. A. Science 2004, 306, 666.

[2] Hu, Y. J.; Jin, J.; Zhang, F.; Wu, P.; Cai, C. X. Acta Phys.-Chim. Sinica 2010, 26, 2073 (in Chinese). (胡耀娟，金娟，张卉，吴萍，蔡称心， 物理化学学报, 2010, 26, 2073.)

[3] Fan, S. Q.; Tan, R. X.; Xie, X. M.; Zhang, M. Y.; Huang, Q. Z. New Carbon Mater. 2018, 33, 522 (in Chinese). (樊姝婧，谭瑞轩，谢翔 旻, 张明瑜, 黄启忠, 新型炭材料, 2018, 33, 522.)

[4] Le, M. Q. Int. J. Mech. Mater. Des. 2015, 11, 15.

[5] Li, X. Y.; Wang, J.; Wu, R. J. Chin. J. At. Mol. Phys. 2018, 35, 139 (in Chinese). (李旭艳, 王静, 吴荣, 原子与分子物理学报, 2018 , 35, 139.)

[6] Lee, C.; Wei, X.; Kysar, J. W.; Hone, J. Science 2008, 321, 385.

[7] Chen, J. H.; Jang, C.; Xiao, S.; Ishigami, M.; Fuhrer, M. S. Nat. Nanotechnol. 2008, 3, 206.

[8] Balandin, A. A.; Ghosh, S.; Bao, W.; Calizo, I.; Teweldebrhan, D.; Miao, F.; Lau, C. N. Nano Lett. 2008, 8, 902.

[9] Liu, X. Ph.D. Dissertation, Donghua University, Shanghai, 2016 (in Chinese). (刘霞，博士论文，东华大学，上海, 2016.)

[10] Walker, L. S.; Marotto, V. R.; Rafiee, M. A.; Koratkar, N.; Corral, E. L. ACS Nano 2011, 5, 3182.

[11] Kim, H.; Abdala, A. A.; Macosko, C. W. Macromolecules 2010, 43, 6515.

[12] Bai, H.; Li, C.; Shi, G. Adv. Mater. 2011, 23, 1089

[13] Geng, Z.; Hähnlein, B.; Granzner, R.; Auge, M.; Ledeveb, A. A.; Davydov, V. Y.; Kitller, M.; Pezoldt, J.; Schwierz, F. Ann. Phys. 2017, 529, 1700033.

[14] Wang, H.; Kurata, K.; Fukunaga, T.; Ago, H.; Takamatsu, H.; Zhang, X.; Ikuta, T.; Takahashi, K.; Nishiyama, T.; Takata, Y. Sens. Actuators, $A$ 2016, 247, 24.

[15] Ma, C. M.S. Thesis, Harbin Institute of Technology, Harbin, 2015 (in Chinese). (马聪, 硕士论文, 哈尔滨工业大学, 哈尔滨, 2015.)

[16] Tan, Y. B.; Lee, J. M. J. Mater. Chem. A 2013, 1, 14814.

[17] Liu, Y.; Tao, W.; Wu, D. Chin. J. Chem. 2020, 38, 1123.

[18] Hill, E. W.; Vijayaragahvan, A.; Novoselov, K. IEEE Sens. J. 2011 11,3161

[19] Mackin, C.; Schroeder, V.; Zurutuza, A.; Su, C.; Kong, J.; Swager, T. M.; Palacios, T. ACS Appl. Mater. Interfaces 2018, 10, 16169.

[20] Miao, J.; Shi, Y.; Zhu, H.; Gao, M. Chin. J. Chem. 2020, 38, 719.

[21] Zhang, H.; Gruener, G.; Zhao, Y. J. Mater. Chem. B 2013, 1, 2542.

[22] Byun, J. J. Microbiol. Biotechnol. 2015, 25, 145.

[23] Lu, J.; Tan, S.; Zhu, Y.; Li, W.; Chen, T.; Wang, Y.; Liu, C. Acta Chim. Sinica 2019, 77, 253 (in Chinese). (卢静荷, 谭淑珍, 朱雨清, 李伟, 陈天啸, 王瑶, 刘陈, 化学学报, 2019, 77, 253.)

[24] Song, G.; Wu, T.; Liu, F.; Zhang, B.; Liu, X. Acta Chim. Sinica 2020, 78,82 (in Chinese). (宋光捷, 武调弟, 刘福金金, 张涁雁, 刘秀辉, 化学学报, 2020, 78, 82.) 
[25] Meyer, J. C.; Geim, A. K.; Katsnelson, M. I.; Novoselov, K. S.; Booth, T. J.; Roth, S. Nature 2007, 446, 60.

[26] Sutter, P. W.; Flege, J. I. Nat. Mater. 2008, 7, 406

[27] Emtsev, K. V.; Bostwick, A.; Horn, K.; Jobst, J.; Kellogg, G. L.; Ley, L.; McChesney, J. L.; Ohta, T.; Reshanov, S. A.; Rohrl, J.; Rotenberg, E.; Schmid, A. K.; Waldmann, D.; Weber, H. B.; Seyller, T. Nat. Mater. 2009, 8, 203.

[28] Li, X.; Cai, W.; An, J.; Kim, S.; Nah, J.; Yang, D.; Piner, R.; Velamakanni, A.; Jung, I.; Tutuc, E.; Banerjee, S. K.; Colombo, L.; Ruoff, R. S. Science 2009, 324, 1312.

[29] Chae, S. J.; Güneş, F.; Kim, K. K.; Kim, E. S.; Han, G. E.; Kim, S. M.; Shin, H.; Yoon, S.; Choi, J.; Park, M. H.; Yang, C. W; Pribat, D.; Lee, Y. H. Adv. Mater. 2009, 21, 2328.

[30] Zhu, Y.; Stoller, M. D.; Cai, W.; Velamakanni, A.; Piner, R. D.; Chen. D. Ruoff, R. S. ACS Nano 2010, 4, 1227.

[31] Chen, W.; Yan, L. Nanoscale 2010, 2, 559.

[32] Hernandez, Y.; Nicolosi, V.; Lotya, M.; Blighe, F. M.; Sun, Z.; De, S.; McGovern, I. T.; Holland, B.; Byrne, M.; GunKo, Y. K.; Boland, J. J.; Niraj, P.; Duesberg, G.; Krishnamurthy, S.; Goodhue, R.; Hutchison, J.; Scardaci, V.; Ferrari, A. C.; Coleman, J. N. Nat. Nanotechnol. 2008, 3, 563

[33] Hernandez, Y.; Lotya, M.; Rickard, D.; Bergin, S. D.; Coleman, J. N. Langmuir 2010, 26, 3208.

[34] Lotya, M.; Hernandez, Y.; King, P. J.; Smith, R. J.; Nicolosi, V.; Karlsson, L. S.; Blighe, F. M.; De, S.; Wang, Z.; McGovern, I. T.; Duesberg, G. S.; Coleman, J. N. J. Am. Chem. Soc. 2009, 131, 3611.

[35] Coleman, J. N. Adv. Funct. Mater. 2009, 19, 3680.

[36] Coleman, J. N. Acc. Chem. Res. 2013, 46, 14.

[37] Shih, C. J.; Lin, S.; Strano, M. S.; Blankschtein, D. J. Am. Chem. Soc. 2010, 132, 14638.

[38] Fu, C.; Yang, X. Carbon 2013, 55, 350.

[39] Yang, J.; Yang, X.; Li, Y. Curr. Opin. Colloid Interface Sci. 2015, 20, 339.

[40] Xu, X.; Cai, L.; Zheng, X.; Xu, Q. Phys. Chem. Chem. Phys. 2017, 19,16062

[41] Chiu, P. L.; Mastrogiovanni, D. D. T.; Wei, D.; Louis, C.; Jeong, M.; Yu, G.; Saad, P.; Flach, C. R.; Mendelsohn, R.; Garfunkel, E.; He, H. J. Am. Chem. Soc. 2012, 134, 5850.
[42] Shi, M. Y.; Zhang, X. F.; Wang, X. Y.; Wang, W. Z.; Jiang, X. Q. J. Nanjing Norm. Univ. (Engineering and Technology) 2014, 14, 1 (in Chinese). (石梦燕, 张晓凤, 王孝英, 王文珠, 蒋晓青, 南京师范 大学学报: 工程技术版, 2014, 14, 1.)

[43] Chen, J.; Shi, W.; Gao, Z.; Wang, T.; Wang, S.; Dong, L.; Yang, Q.; Xiong, C. Nano Res. 2018, 11, 820.

[44] Kim, H. S.; Oweida, T. J.; Yingling, Y. G. J. Mater. Sci. 2018, 53 , 5766.

[45] Kim, H. S.; Huang, S. M.; Yingling, Y. G. MRS Adv. 2016, 1, 1883.

[46] Kim, H. S.; Farmer, B. L.; Yingling, Y. G. Adv. Mater. Interfaces 2017, 4, 1601168

[47] Sun, J.; Li, Y.; Lin, J. J. Mol. Graphics Modell. 2017, 74, 16.

[48] da Silva, A. W. S.; Vranken, W. F. BMC Res. Notes 2012, 5, 367

[49] Mandell, M. J.; McTague, J. P.; Rahman, A. J. Chem. Phys. 1976, 64, 3699.

[50] Hess, B.; Bekker, H.; Berendsen, H. J. C.; Fraaije, J. G. E. M. J. Comput. Chem. 1997, 18, 1463.

[51] Hess, B. J. Chem. Theory Comput. 2008, 4, 116.

[52] Darden, T.; York, D.; Pedersen, L. J. Chem. Phys. 1998, 98, 10089.

[53] Essmann, U.; Perera, L.; Berkowitz, M. L.; Darden, T.; Lee, H.; Pedersen, L. G. J. Chem. Phys. 1995, 103, 8577.

[54] Yau, A. W.; Pritchard, H. O. Can. J. Chem. 1977, 55, 992.

[55] Berendsen, H. J. C.; Postma, J. P. M.; van Gunsteren, W. F.; DiNola, A.; Haak, J. R. J. Chem. Phys. 1984, 81, 3684.

[56] Bussi, G.; Donadio, D.; Parrinello, M. J. Chem. Phys. 2007, 126, 14101.

[57] Parrinello, M.; Rahman, A. J. Appl. Phys. 1981, 52, 7182.

[58] Hoover, W. G. Phys. Rev. A 1985, 31, 1695.

[59] Jussila, H.; Yang, H.; Granqvist, N.; Sun, Z. Optica 2016, 3, 151.

[60] Wolfe, M.; Jonas, J. J. Chem. Phys. 1979, 71, 3252.

[61] Lide, D. R. CRC handbook of chemistry and physics: a ready-reference book of chemical and physical data, CRC Press, New York, 1995.

[62] Carugo, O.; Pongor, S. Protein Sci. 2001, 10, 1470.

[63] Moghaddam, M. B.; Goharshadi, E. K.; Entezari, M. H.; Nancarrow, P. Chem. Eng. J. 2013, 231, 365 .

[64] Li, B.; Hong, S.; Zhang, X.; Xiong, C.; Zhao, G.; Yang, Q.; Liu, H. Adv. Theory Simul. 2019, 2, 1900155. 